Recepción: 15 / 04 / 2017

Ciencias Informáticas

Aceptación: 01 / 05 / 2017

Revisión de Literatura

Publicación: 15 / 05 / 2017

\title{
Tecnología Wi-Fi para cubrir déficit de telefonía fija en zonas rurales
}

\author{
Wi-Fi technology to cover fixed telephony deficit in rural areas
}

\section{A tecnologia Wi-Fi para cobrir déficits de telefonia fixa em áreas rurais}

\author{
Eduardo A. Alvarado-Unamuno \\ eduardo.alvaradou@ug.edu.ec \\ Luís A. Choez-Ruiz" \\ luis.choezr@ug.edu.ec \\ Josué R. Pérez-Sánchez "' \\ josue.perezs@ug.edu.ec
}

Correspondencia: eduardo.alvaradou@ug.edu.ec

\footnotetext{
Magister en Sistemas de Información Gerencial; Diploma Superior en Docencia y Evaluación de la Educación Superior; Ingeniero en Electricidad Especialización Electrónica, FCMF, CINT. Universidad de Guayaquil, Ecuador. Ingeniero en Networking y Telecomunicaciones, Universidad de Guayaquil, Ecuador. Ingeniero en Networking y Telecomunicaciones, Universidad de Guayaquil, Ecuador.
} 


\section{Resumen}

En la actualidad existen zonas rurales y urbanas en el Ecuador donde la única opción para brindar el servicio de telefonía es la telefonía celular o en el caso más crítico no cuenta con ningún servicio de telecomunicaciones, considerando a estas zonas como áreas de necesidad prioritaria de acceso a servicios de telecomunicaciones. Una de las causas por las que se produce ésta situación son las características geográfica de la zona, costo de implementación y el despliegue de la red de abonados. El objetivo del presente trabajo de investigación es demostrar la factibilidad de la utilización de la tecnología Wi-Fi para cubrir las necesidades de telefonía fija en zonas rurales. La investigación parte de los datos proporcionados por las entidades gubernamentales encargadas del control y regulación de este servicio. Se presenta el análisis de la aplicación de las redes inalámbricas Wi-Fi en combinación con sistemas de software libre y su aplicación en una zona rural de la costa ecuatoriana. Se concluye que es factible la utilización de la tecnología Wi-Fi para brindar servicios de telefonía fija.

Palabras clave: Wi-Fi; VoWLAN; Telefonía Fija. 


\begin{abstract}
At present there are rural and urban areas in Ecuador where the only option to provide the telephone service is cellular telephony or in the most critical case they do not have any telecommunications service, considering these areas as priority areas of access To telecommunications services. One of the causes of this situation is the geographical characteristics of the area, cost of implementation and the deployment of the subscriber network. The aim of this research is to demonstrate the feasibility of using Wi-Fi technology to meet the needs of fixed telephony in rural areas. The investigation starts from the data provided by the governmental entities in charge of the control and regulation of this service. The analysis of the application of wireless networks in combination with free software systems and their application in a rural area of the Ecuadorian coast is provided in this study. It is concluded that it is feasible to use Wi-Fi technology to provide fixed telephony services.
\end{abstract}

Key words: Wi-Fi; VoWLAN; Fixed Telephony. 


\section{Resumo}

Atualmente existem áreas rurais e urbanas no Equador onde a única opção para prestar o serviço telefônico é a telefonia celular ou no caso mais crítico não têm qualquer serviço de telecomunicações, considerando essas áreas como áreas prioritárias de acesso aos serviços de telecomunicações. Uma das causas desta situação são as características geográficas da área, o custo de implementação ea implantação da rede de assinantes. O objetivo desta pesquisa é demonstrar a viabilidade da utilização da tecnologia Wi-Fi para atender as necessidades de telefonia fixa nas áreas rurais. A investigação parte dos dados fornecidos pelas entidades governamentais responsáveis pelo controle e regulação deste serviço. A análise da aplicação de redes sem fio em combinação com sistemas de software livre e sua aplicação em uma área rural da costa equatoriana é fornecida neste estudo. Conclui-se que é viável utilizar a tecnologia Wi-Fi para fornecer serviços de telefonia fixa.

Palavras chave: Wi-fi; VoWLAN; telefonia fixa. 


\section{Introducción.}

En el Ecuador, la era innovadora de las telecomunicaciones se inicia en Octubre de 1972 cuando se crea el Instituto Ecuatoriano de Telecomunicaciones (IETEL), luego transcurren 20 años sin cambio alguno en el marco regulatorio. En el año 1992 se expide la Ley Especial de Telecomunicaciones y se determina que los servicios básicos de telecomunicaciones son brindados por el Estado a través de la Empresa Estatal de Telecomunicaciones (EMETEL) que reemplazó a IETEL. Las leyes de telecomunicaciones hasta el año 2000 sufrieron una serie de reformas que en el mes de Marzo fueron declaradas dentro del Régimen de Libre Competencia en las Telecomunicaciones y para el año siguiente se da el instrumento legal que regula con detalle la libre competencia en las telecomunicaciones ecuatorianas, [1] esto permitió que se incrementen el número de empresas que pueden ofrecer servicios de telecomunicaciones en el Ecuador.

Los servicios de telecomunicaciones que pueden ofrecer las empresas en el Ecuador están definidos en la Ley Orgánica de Telecomunicaciones (LOT) en su artículo 36, los define como aquellos servicios que se soportan sobre redes de telecomunicaciones con el fin de permitir y facilitar la transmisión y recepción de signos, señales, textos, vídeo, imágenes, sonidos o información de cualquier naturaleza, para satisfacer las necesidades de telecomunicaciones de los abonados, clientes, usuarios. Dentro de los servicios de telecomunicaciones en forma ejemplificativa y no limitativa, se citan a la telefonía fija y móvil, portadores y de valor agregado [2].

Por otro lado, los servicios de valor agregado se definen como aquellos que utilizan servicios finales de telecomunicaciones (SMA, Telefonía Fija) y/o servicios portadores de telecomunicaciones para llegar a sus usuarios finales, e incorporan aplicaciones que permiten transformar el contenido de la información trasmitida. Ejemplo de servicio de valor agregado es el acceso a Internet [3]. 
Las mayores empresas del sector de las telecomunicaciones, invierten en el fortalecimiento de sus redes para dar mayor cobertura a los usuarios en los sectores urbanos de las ciudades más representativas ya que en ellas se encuentran la mayor cantidad de usuarios que demandan de sus servicios y tienen el poder adquisitivo para pagarlos. Por el contrario, en determinadas zonas rurales donde la cantidad de usuarios es limitado dado a su situación económica para solicitar servicios de telecomunicaciones es pequeño, que a su vez los que instan en el servicio no son atendidos de manera satisfactoria.

Para estas empresas las zonas rurales no son su mayor enfoque si de economía se trata, ya que los bajos ingresos por la prestación de sus servicios versus los costos altos de implementación y despliegue de sus redes de telecomunicaciones, no los hace rentables, y consideran internamente un gasto innecesario.

En Latino América existe abundante evidencia de microempresas proveedoras de servicios de telecomunicaciones que atienden zonas rurales de escaso interés para las grandes empresas proveedoras de servicios, brindando servicios con precios asequibles permitiendo a sus habitantes integrarse a la sociedad digital del conocimiento.

La empresa TelecomSystem brinda servicios de acceso Internet a la comuna Bajada de Chanduy, cercana a Cerecita Via Guayaquil - Salinas, ubicado en la Provincia de Santa Elena con una población aproximadamente de 1750 habitantes, cuenta con más de 250 clientes residenciales y corporativos suscritos al servicio de banda ancha ilimitada, también cuenta con tres sucursales dentro de la costa Ecuatoriana, ofreciendo sus servicios a los habitantes, dueños de haciendas y camaroneras del sector y así promueven servicios TIC en sectores alejados de las grandes urbes [4]. 
En esta zona rural, no existe otro proveedor que ofrezca a los moradores del sector este beneficio de comunicación, sin embargo esta empresa no brinda el servicio de telefonía análoga o digital a sus subscriptores, los usuarios acceden a los servicios de telefonía, a través de la telefonía móvil celular la cual no cuenta con una buena cobertura en la zona que genera malestar en los usuarios. La demanda que tienen los suscriptores de servicios de comunicación, como es la telefonía fija convencional conlleva a la necesidad de implementar una central Telefónica IP.

El propósito de este estudio, es un análisis de caso de una microempresa proveedora de servicios de valor agregado en la zona rural del Guayas, con una alternativa tecnológica viable para cubrir las necesidades de telefonía fija en esta zona rural, específicamente utilizando tecnología WiFi.

\section{Análisis teórico}

\section{Redes Inalámbricas}

Las redes inalámbricas basada en el estándar IEEE802.11 (también llamado Wi-Fi) en la actualidad son ampliamente utilizadas, aunque estas redes no fueron creadas para transportar aplicaciones en tiempo real como la voz, su amplia disponibilidad y bajos costos las convierten en una solución atractiva para añadir la movilidad a la Voz sobre IP (VoIP), la combinación de VoIP y Wi-Fi (VoWi-Fi), ha generado gran interés [5].

Sin embargo, existen varios impedimentos técnicos que se deben superar en el estándar IEEE802.11 para implementar Voz, uno de ellos es la falta de calidad de servicio (QoS), la UIT-T G.114 recomienda que el retardo de un paquete de voz unidireccional debe ser inferior a $150 \mathrm{mseg}$, debido a que 150 mseg es el retardo del camino de extremo a extremo, lo cual significa que el 
acceso al canal LAN inalámbrico debe ser considerablemente inferior a 150 ms, también algunos códec como el G.729 soporta una pérdida de paquete menor al 1\% para prevenir errores audibles. [6]

Se ha realizado análisis en redes inalámbricas al ejecutar aplicaciones de tiempo real como la voz, como ejemplo se ha implementado mecanismos de Backoff Control y Priority Queuing (BCPQ) en el punto de acceso (AP) este mecanismo mejora notablemente el rendimiento de la voz [7], también el desarrollo de Software basado en Time-Division Multiple Access (STDMA) que garantiza QoS por conexión, así como la mejora en eficiencia de utilización del canal de radio.[6]

Con el continuo desarrollo de las redes Wireless (WLAN) se han propuesto varias extensiones del estándar IEEE802.11 para mejorar su capacidad de manejar aplicaciones de tiempo real, tanto así que hoy los proveedores de servicio de telefonía celular ven a la VoWi-Fi como una opción viable para sus redes de Voz [8].

Voz sobre IP

Las telecomunicaciones han ido evolucionando a lo largo de la historia, desde los servicios de telefonía dados por las PSTN tradicionales con teléfonos análogos hasta las grandes redes de datos como la Internet. La convergencia entre las redes de voz, datos y video han revolucionado las telecomunicaciones en el mundo entero permitiendo una comunicación enriquecida en el Internet entre dos o más usuarios remotos.

Las implementaciones iniciales de la tecnología Voz sobre IP denominadas VoIP fueron basados inicialmente en protocolos propietarios y luego el desarrollo hacia una interoperabilidad dieron paso a estándares abiertos como H323 y MEGACO. Siendo el protocolo H.323 del UIT-T el 
más ampliamente adoptado. H.323 ofrece una solución completa para el establecimiento y control de llamadas, pero puede que no sea adecuado en ciertos escenarios [9].

El protocolo de inicio de sesión (SIP) de la IETF tiene un enfoque más flexible que permite su uso potencial en muchas aplicaciones. SIP es protocolo de señalización de capa de aplicación que se utiliza para establecer y finalizar sesiones multimedia tales como llamadas VoIP, las comunicaciones que utilizan SIP están basada en texto y consta de solicitudes y respuestas. En su forma más simple, SIP puede trabajar bajo un modelo peer-to-peer donde un usuario envía una invitación directa a otro usuario para establecer una llamada. El protocolo SIP está siendo adoptado cada vez más en aplicaciones como por ejemplo VoIP, mensajería instantánea, videoconferencia, entre otros [9].

Asterisk [10] es un software de código abierto (open source) también llamado software libre que permite implementar funcionalidades avanzadas de una central telefónica privada (PBX), ofrece VoIP y soporta múltiples protocolos tales como SIP, H323 y AIX, también existen soluciones de PBX no gratuitas las cuales en ocasiones son propietarias y no son muy flexibles.

\section{Telefonía fija en Ecuador}

En el Ecuador, se estableció el Plan Nacional de Telecomunicaciones y Tecnologías de Información del Ecuador 2016 - 2021[11] por el el Ministerio de Telecomunicaciones (MINTEL), este es el instrumento de planificación y gestión del sector de las telecomunicaciones y tecnologías de la información cuyo objetivo es conseguir una mayor inclusión digital y competitividad del país. 
Este plan cuenta con macro-objetivos entre los cuales se menciona el completar y fomentar el despliegue de infraestructura de telecomunicaciones, aumentar la penetración de servicios TIC en la población y asegurar el uso de las TIC para el desarrollo socio-económico. [11] .

La telefonía fija entre los años 2007 y 2014 dio como resultado un incremento del $1.85 \%$ [12], según el ARCOTEL, la telefonía fija tiene un bajo crecimiento y una concentración de despliegue de infraestructura en las grandes ciudades y con el fin de atender los sectores sociales menos favorecidos que se encuentran ubicados en las áreas urbanas, urbano marginales y rurales. Se ha establecido en los contratos de concesión de servicios de telefonía fija que los prestadores deben obligadamente mostrar un plan anual de expansión que permitirá seguir ampliando el desarrollo de la telefonía fija y su cobertura a nivel nacional.

De acuerdo a los datos del ARCOTEL en el 2016, la participación en el mercado de la telefonía fija se muestra en la Figura 1, donde se observa que CNT EP y Etapa operadores del estado cubren el $91.25 \%$ y los operadores privados Ecuador Telecom S.A., Level 3 Ecuador S.A. y Linkotel S.A. cubren el 8.51\% [13].

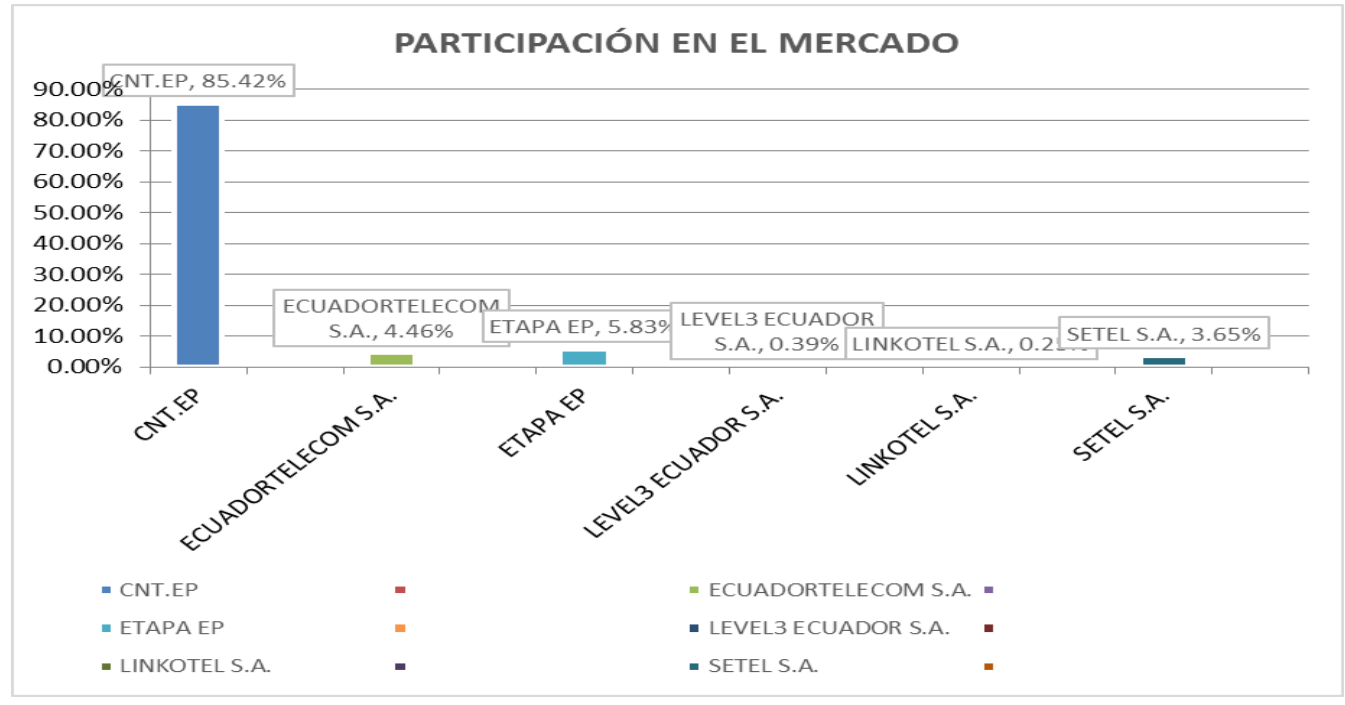

Figura $N^{\circ}$ 1.- Participación en el mercado de los operadores de telefonía fija. 
También indica el ARCOTEL que el número de empresas que dan servicio de acceso a internet ha tenido un incremento basados en el año 1998 que va de 14 a 292 en el 2015, aproximadamente veintiún veces a nivel nacional [12]. Debido al alto número de proveedores existe una alta competencia en cuanto a precios, calidad, garantía de servicio, entre otros.

Un factor a tomar en cuenta por las empresas que desean brindar los servicios de telecomunicaciones, es lo dispuesto en la LOT en su artículo 18 donde se indica que el espectro radioeléctrico es propiedad del estado, su uso y explotación requiere el otorgamiento previo de un título habilitante emitido por la ARCOTEL [2].

Se tiene como antecedente un trabajo del modelo de negocio para la expansión de los servicios de telefonía en los sectores urbanos y rurales del cantón Babahoyo [14] utilizando tecnología inalámbrica Wimax, en donde se concluye que es un modelo rentable y económicamente factible implementar telefonía fija e internet a un costo de aproximadamente de 3.4 millones de dólares con la instalación de 7 radio bases Wimax.

\section{Metodología}

El proyecto de investigación se ubica en la comuna Bajada de Chanduy, en la Figura $\mathrm{N}^{\circ} 2$ se indica la ubicación de la comuna y la torre principal para brindar el servicio de Internet. 


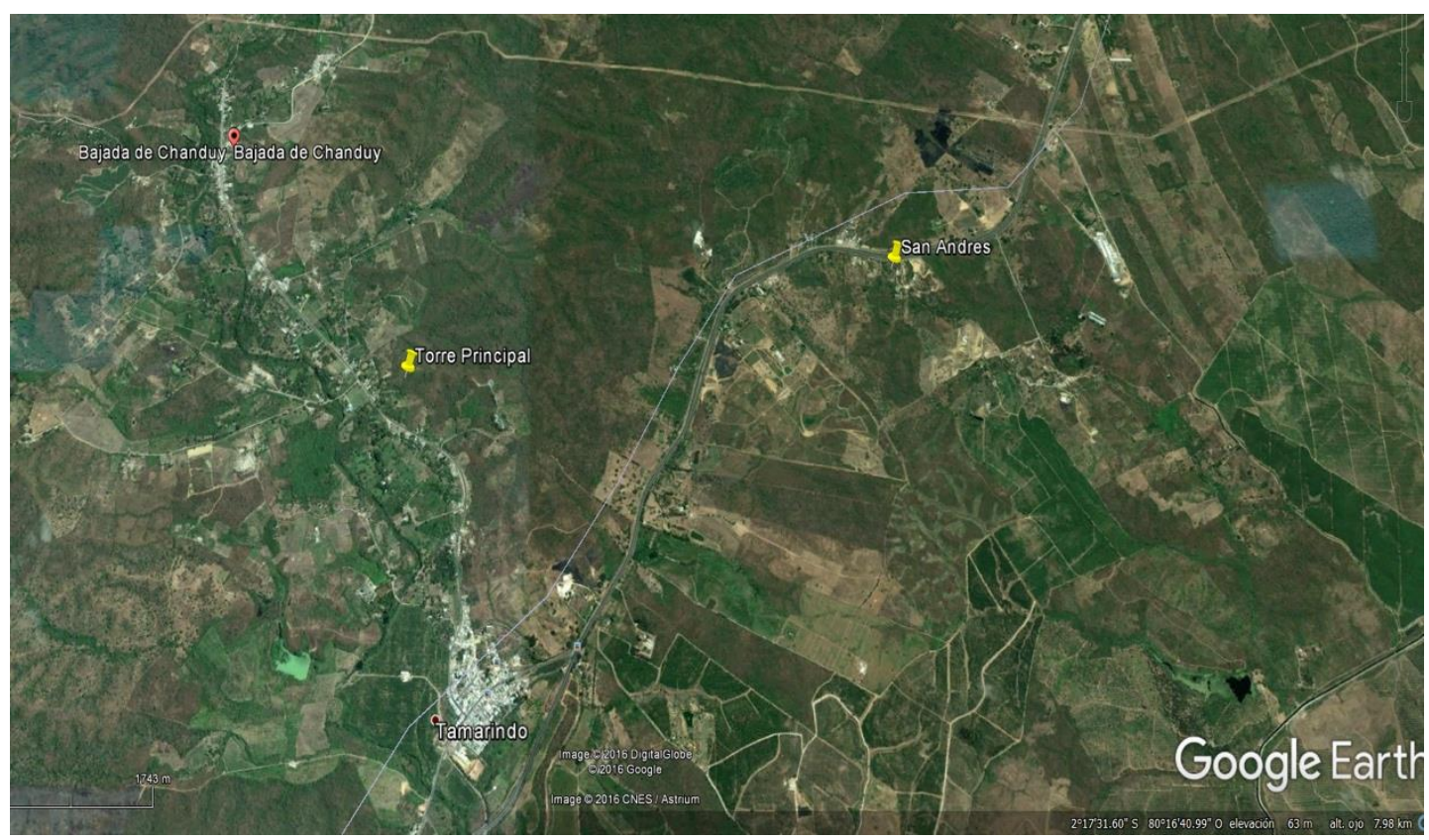

Figura $N^{\circ}$ 2.- Comuna Bajada de Chanduy

El trabajo comprende en el análisis de la situación actual de los servicios de telecomunicaciones que la compañía TelecomSystem brinda en el sector de estudio y el diseño de la infraestructura necesaria para implementar la telefonía fija. Por ello, para poder dar la cobertura a la zona de la comuna el proveedor utilizó una torre de 24 m sobre una elevación de 60 m lo que da una altura total de $84 \mathrm{~m}$.

La red opera en la banda de los $5 \mathrm{Ghz}$ que es una banda no licenciada, para evitar interferencias, el fabricante Mikrotik ha desarrollado el protocolo Nv2, el mismo está basado en la tecnología de acceso al medio TDMA (Acceso Múltiple por División de Tiempo) en lugar de CSMA (Acceso Múltiple por Detección de Portadora), usado en los dispositivos regulares 802.11. que mejora y hace más eficiente el uso del canal, además de mejorar el throughput y la latencia, especialmente en redes PtMP (Punto a Multipunto) [15].

A continuación en la figura 3 se muestra el diagrama de alto nivel de la red de TelecomSystem el proveedor solo brinda el servicio de internet, e inicialmente para el montaje de 
esta infraestructura sin considerar los CPE se incurrió en un gasto de \$ 3.160,00 dólares.

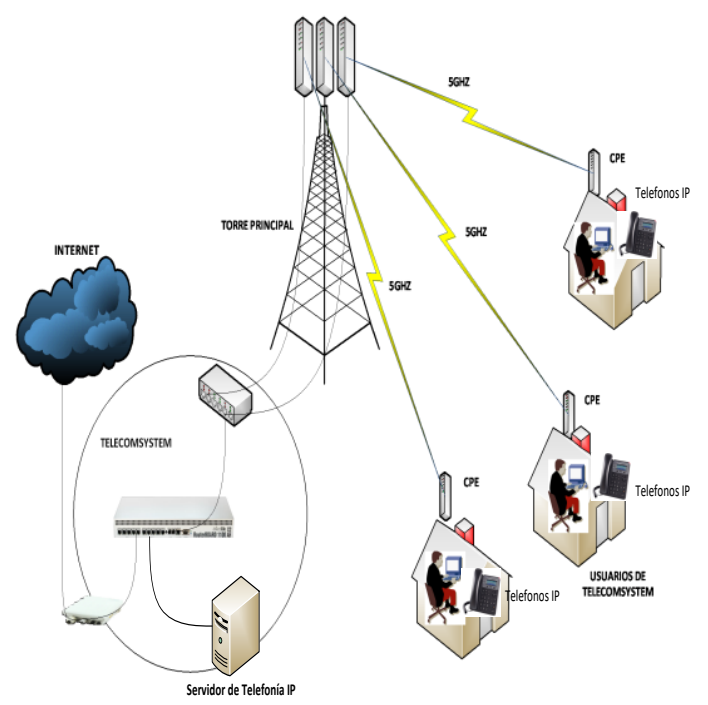

\section{Figura $N^{\circ}$ 3.- Diagrama de Red de TelecomSystem}

Para gestión y administración del ancho de banda se instaló un equipo routerboard AX1100 marca Mikrotik, que es el encargado del control de los clientes, asignando el ancho de banda para cada uno de ellos y mostrara el consumo, tiempo de conexión, y mostrará el resumen de los usuarios conectados en el transcurso del día.

En la torre se instaló 3 antenas sectoriales de $120^{\circ}$ y a cada una de ellas se conectó el equipo BASE BOX 5 de marca Mikrotik con capacidad para 60 CPE.

Del lado de los usuarios se instala el equipo RB912UAG-5HPnD-OUT de marca Mikrotik, la cual tiene un router integrado y un puerto FastEhernet para la conexión de los usuarios.

A la infraestructura indicada se le añadió el servicio de telefonía IP a través de es un servidor con el software de Asterisk, en un esquema inicial se planteó un escenario de pruebas de 5 usuarios de telefonía. Los componentes añadidos a la infraestructura actual los cuales son el servidor de telefonía IP y los teléfonos IP. 
El servidor de telefonía IP se conectó a través de un enrutador a la red cableada, esto se realizó para poder brindar seguridad informática, ya que al estar conectada a la red IP podría ser objeto de ataques informáticos como, por ejemplo, ataques de denegación de servicios.

\section{Resultados}

Para evaluar el diseño tecnológico se elaboró un banco de pruebas que permitió establecer la funcionalidad de la solución de telefonía IP brindada. Se evaluaron funcionalidades como calidad de la voz, identificador de llamada, correo de voz y llamadas en espera que son las funcionalidades básicas que dan los operadores de telefonía fija. Se utilizó Asterisk como servidor SIP y PBX, este software es fácil de configurar y administrar, la PBX y el servidor SIP se ejecutó en equipo con un CPU Intel core I3 de $3.70 \mathrm{Ghz}$ y 4 GB de Ram, en el mismo se mantuvieron los procesos indicados en la Figura 4 [4].

Servidor de telefonía.

Servidor de fax.

Servidor de Correo

Servidor de Base de Datos

Servidor Web

\begin{tabular}{|c|c|c|c|}
\hline \multicolumn{3}{|c|}{ : Estado de Procesos } & \multirow{2}{*}{$\frac{\sqrt{3}}{\nabla}$} \\
\hline d & Servidor Telefónico & ACTIVO & \\
\hline 8 & Servidor de Mensajería Instantánea & INACTIVO & $\nabla$ \\
\hline 口 & Servidor de Fax & ACTIVO & $\nabla$ \\
\hline$\leftarrow$ & Servidor de Correo & ACTIVO & $\nabla$ \\
\hline 8 & Servidor de Base de Datos & ACTIVO & $\nabla$ \\
\hline 8 & Servidor Web & ACTIVO & $\nabla$ \\
\hline G & Servidor CallCenter Elastix & NO INSTALADO & $\nabla$ \\
\hline
\end{tabular}

Figura $N^{\circ}$ 4.-. Procesos de Telefonía instalados en Servidor de PBX. 
La telefonía brindada en las pruebas fue la telefonía fija, por lo tanto se evita los problemas asociados con la movilidad de los usuarios, se evidenció el poco consumo de recursos que puede demandar el establecer una llamada telefónica Figura 5, el uso de CPU no excede del 3\% y el consumo de memoria se mantiene en aproximadamente $320 \mathrm{MB}$ [4].

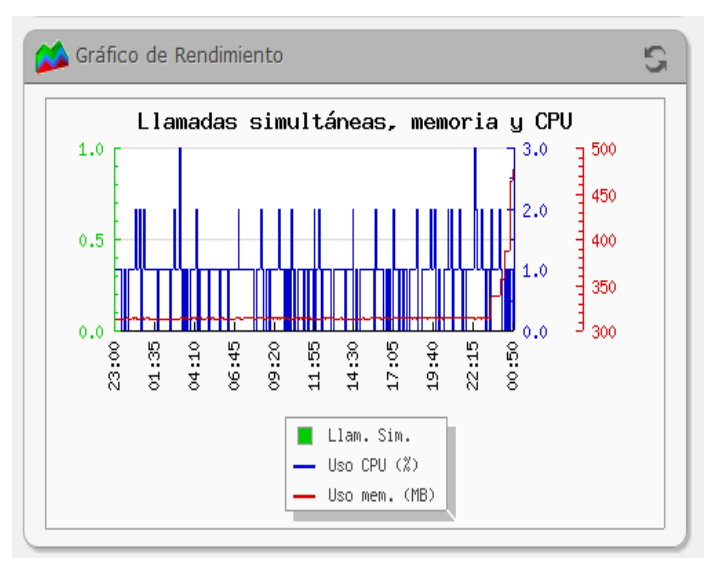

Figura $N^{\circ}$ 5.- Gráfico de consumo de recursos de una llamada VoIP.

Dentro de las pruebas no se incluyó conexiones con la PSTN, dado que era un ambiente de factibilidad técnica de desplegar servicios de telefonía fija utilizando VoWi-Fi y principalmente se requiere que el proveedor tenga el título habilitante de proveedor de servicio de telefonía fija en la zona donde se realizaron las pruebas y actualmente el proveedor no las posee.

Las pruebas de las funcionalidades de la central telefónica fueron satisfactorias para los usuarios ya que podían realizar llamadas entre ellos, los usuarios que tenían internet lograron acceder a través de un navegador a su lista de contacto (guía telefónica virtual de la comunidad), escuchar su correo de voz, identificar la llamada entrante, y se mantuvo una buena calidad de llamada. 
La implementación de este sistema permite integrar a esta comuna ya que mantendrá a sus habitantes comunicados con sus familiares, amigos y en general con la sociedad ecuatoriana. En general, es satisfactorio el resultado de esta primera etapa, ya que no se está considerando el tema de seguridad informática a profundidad, lo cual puede generar un segundo estudio, porque estos sistemas al estar en red son propensos a ser atacados.

\section{Conclusiones}

El propósito de este estudio, es un análisis de caso de una microempresa proveedora de servicios de valor agregado en la zona rural del Guayas, y presentar una alternativa tecnológica viable para cubrir las necesidades no atendidas de servicios de telecomunicaciones en esta zona rural, específicamente utilizando tecnología Wi-Fi para brindar servicios de telefonía fija.

En el presente trabajo de investigación se implementó el servicio de telefonía fija utilizando la infraestructura actual del proveedor de internet solo agregando un servidor, los teléfonos IP y la debida configuración, con lo que se demuestra que la implementación de telefonía fija es fácil y demanda pocos recursos en microempresas proveedoras de internet.

Los sistemas que utilizan Wi-Fi en el acceso permiten un rápido despliegue de las redes de los proveedores de servicios en zonas rurales a bajos costos, si bien es cierto Wi-Fi no fue pensada en aplicaciones de tiempo real como la voz, el desarrollo de nuevas extensiones al estándar IEEE 802.11 y protocolos propietarios lo hacen totalmente viable para el despliegue de VoWi-Fi.

En el Ecuador y en la provincia del Guayas se convierte en una alternativa totalmente viable que pueden utilizar las micro empresas proveedores de servicios, para cumplir con lo indicado por los organismos de control ARCOTEL y MINTEL en el sentido de completar y 
fomentar el despliegue de infraestructura de telecomunicaciones, aumentar la penetración de servicios TIC en la población y asegurar el uso de las TIC para el desarrollo económico y social en las zonas rurales.

\section{Agradecimientos.}

A la empresa TelecomSystem, a través de su representante el Ing. David Cedeño Alvarado por ceder sus instalaciones para el desarrollo de las pruebas.

A la licenciada Viviana Pinos por su colaboración en la revisión del documento.

\section{Bibliografía.}

[1] R. Barrantes and P. Pérez, "Regulación e Inversión en Telecomunicaciones Estudio de caso para el Perú: Setiembre 2006-Agosto 2007," OSIPTEL, Perú, pp. 1-60, 2007.

[2] Asamblea Nacional del Ecuador, "Ley Organica de Telecomunicaciones," p. 55, 2015.

[3] "Servicios de Valor Agregado (Internet y otros) | Agencia de Regulación y Control de las Telecomunicaciones | Ecuador." [Online]. Available: http://www.arcotel.gob.ec/servicios-de-valoragregado-internet-y-otros/. [Accessed: 16-Mar-2017].

[4] L. Choez and J. Perez, "“implementacion de telefonia ip con elastix para un isp con acceso wifi,"” 2016.

[5] S. Act, "Ready for," New York, no. January, pp. 19-24, 2006.

[6] F. Guo and T. Chiueh, "Comparison of QoS guarantee techniques for VoIP over IEEE802.11 wireless LAN,” Proc. SPIE, vol. 6818, p. 68180J-68180J-12, 2008.

[7] F. Anjum et al., "Voice performance in WLAN networks - an experimental study," GLOBECOM '03. IEEE Glob. Telecommun. Conf. (IEEE Cat. No.03CH37489), vol. 6, pp. 3504-3508, 2003.

[8] S. Fabrice, M. Ngongang, N. Tadayon, and G. Kaddoum, "Voice over Wi-Fi : Feasibility Analysis," pp. 133-138, 2016.

[9] L. Lambrinos, "Deploying open source IP telephony in rural environments," Proc. - 2nd Int. Conf. Next Gener. Mob. Appl. Serv. Technol. NGMAST 2008, pp. 623-627, 2008.

[10] "Open Source Communications Software | Asterisk Official Site." [Online]. Available: http://www.asterisk.org/. [Accessed: 17-Mar-2017].

[11] MINTEL, "Plan Nacional de Telecomunicaciones y Tecnologias de Informacion del Ecaudor 2016 - 
2021."

[12] AGENCIA DE REGULACION Y CONTROL DE LAS TELECOMUNICACIONES, "Boletin Estadictico del Sector de Telecomunicaciones $\mathrm{N}^{\circ} 4 . "$

[13] AGENCIA DE REGULACION Y CONTROL DE LAS TELECOMUNICACIONES, "Servicio de Telefonía Fija | Agencia de Regulación y Control de las Telecomunicaciones | Ecuador." [Online]. Available: http://www.arcotel.gob.ec/servicio-de-telefonia-fija/. [Accessed: 28-Mar-2017].

[14] D. M. Parra, E. N. Morillo, and J. L. Torres, "MODELO DE NEGOCIO PARA LA EXPANSIÓN DE LOS SERVICIOS DE TELEFONÍA FIJA Y DE INTERNET EN LOS SECTORES URBANO Y RURALES DEL CANTÓN BABAHOYO.”

[15] Mikrotik, "Nv2 spanish - MikroTik Wiki." [Online]. Available: https://wiki.mikrotik.com/wiki/Nv2_spanish. [Accessed: 26-Mar-2017]. 